'Escuela de Medicina. Facultad de Medicina, Pontificia Universidad Católica de Chile. Santiago, Chile. ${ }^{2}$ Departamento de Salud Pública. Facultad de Medicina, Pontificia Universidad Católica de Chile. Santiago, Chile. ${ }^{3}$ Departamento de Estadística, Facultad de Matemáticas, Pontificia Universidad Católica de Chile. Santiago, Chile. ${ }^{4}$ Departamento de Gastroenterología. Facultad de Medicina, Pontificia Universidad Católica de Chile. Santiago, Chile. aAlumno de Medicina. bestadístico.

Recibido el 9 de abril de 2014 aceptado el 16 de diciembre de 2014

Conflictos de intereses: ninguno que declarar.

Apoyo financiero: La Encuesta Nacional de Salud 2009-2010 fue financiada por el Ministerio de Salud y la Facultad de Medicina de la Pontificia Universidad Católica de Chile. El módulo digestivo de la ENS 2009-2010 recibió apoyo del Proyecto FONDECYT 1130303 de JFM.

Correspondencia a: Dr. Gonzalo Valdivia Cabrera Departamento de Salud Pública. Facultad de Medicina, Pontificia Universidad Católica de Chile. Marcoleta 434, Santiago Casilla $114 \mathrm{D}$

Teléfono/Fax: + 562-23543038 $+562-26331840$ valdivia@med.puc.cl.

\section{Cobertura de la estrategia preventiva de cáncer de vesícula biliar en Chile: Resultados de la Encuesta Nacional de Salud 2009-2010}

\author{
GONZALO LATORRE S. ${ }^{1}$, DANISA IVANOVIC-ZUVIC S. ${ }^{1}$, \\ ÓSCAR CORSI S. ${ }^{1, a}$, GONZALO VALDIVIA C. ${ }^{2}$, \\ PAULA MARGOZZINI M. ${ }^{2}$, RICARDO OLEA O., ${ }^{3, b}$ \\ JOSÉ CHIANALE B. ${ }^{4}$, JUAN FRANCISCO MIQUEL P. ${ }^{4}$
}

\section{Coverage of the gallbladder cancer prevention strategy in Chile: results from the 2009-2010 National Health Survey}

Background: In Chile, gallbladder cancer (GBC) is one of the most important causes of death and gallstone disease (GSD) is its main risk factor. Abdominal ultrasonography (AU) is used for the diagnosis of GSD and cholecystectomy is used to prevent it. Aim: To estimate GSD prevalence in the general population and to assess the diagnostic and therapeutic coverage of GSD as a preventive strategy for GBC in Chile. Material and Methods: A standardized digestive symptoms questionnaire of the 2009-2010 Chilean National Health Survey was answered by 5412 adults over 15 years old. Self-reports of AU, GBD and cholecystectomies were recorded. Results: The prevalence of biliary-type pain was $7.1 \%$. During the last five years, the prevalence of AU was $16 \%$. GSD was reported in $20 \%$ of these tests and $84 \%$ of them were asymptomatic. The prevalence of $A U$ was significantly lower in Araucania region and among people with less than 12 years of education. Life cholecystectomy prevalence was $11 \%$ and reached $40 \%$ in people aged over 60 years. Women accounted for $75 \%$ of total cholecystectomies. Twenty-one percent of individuals who referred biliary-type pain, were studied with an AU. Only 60\% of people with GSD confirmed by AU underwent a cholecystectomy. Conclusions: GSD affects at least 27\% of the Chilean adult population. Important deficits and inequities in GSD diagnostic and therapeutic coverage were identified.

(Rev Med Chile 2015; 143: 158-167)

Key words: Cholecystectomy; Gallbladder neoplasms; Gallstones; Secondary prevention.
E 1 cáncer vesicular $(\mathrm{CaV})$ en Chile, junto al cáncer de mama, lidera las causas de muerte por cáncer en mujeres y constituyó la cuarta causa de muerte por cáncer en hombres, en el año 2010. En ese año se reportaron 1.751 muertes por $\mathrm{CaV}$ o de la vía biliar en Chile ${ }^{1}$. Presenta una tasa ajustada de mortalidad de $8,9 \times 100.000$ habitantes para ambos sexos, la más alta del mundo ${ }^{2}$, valor que se ha mantenido constante durante la última década. En mujeres, la mortalidad aumenta 7 veces a partir de la cuarta década ${ }^{3}$. La sobrevida depende principalmente del estadio de la enfermedad al momento del diagnóstico ${ }^{4,5}$.

El principal factor de riesgo del $\mathrm{CaV}$ es la cole- 
litiasis $(\mathrm{CL})^{6-8}$. Ésta se reporta en 79 a $100 \%$ de los $\mathrm{CaV}^{9-11}$. En Chile se ha estimado que hasta el 21\% de los hombres y el 50\% de las mujeres mayores de 20 años presentarían CL, alcanzando cerca de 60\% en mujeres a partir de la sexta década ${ }^{11-17}$. Existe también una gradiente litogénica entre etnias, siendo la población mapuche la más afectada ${ }^{15}$, entre otros factores de riesgo descritos ${ }^{7,18-21}$.

El síntoma cardinal de CL es el dolor abdominal de tipo biliar o cólico biliar, el cual aparece en la evolución de la CL aproximadamente en 20\% de los pacientes en países desarrollados y hasta $50 \%$ de los pacientes chilenos ${ }^{16}$. En poblaciones con baja prevalencia de $\mathrm{CaV}$ se ha demostrado que la CL asintomática o silente tiene una historia natural benigna y no se recomienda la colecistectomía preventiva ${ }^{22-24}$. Sin embargo, existe escasa información sobre la historia natural de la CL asintomática en nuestro país ${ }^{25}$.

El método de elección para pesquisar la CL es la ecotomografía abdominal (EA). Ante el hallazgo de CL, la colecistectomía constituye una intervención preventiva para el desarrollo de $\mathrm{CaV}$, con un potencial curativo del $\mathrm{CaV}$ en sus estadios iniciales ${ }^{26,27}$. En nuestro país se ha descrito el hallazgo histopatológico de $\mathrm{CaV}$ entre el $1 \mathrm{al}$ 3,3\% de las colecistectomías en pacientes sintomáticos ${ }^{28-31}$.

No existe evidencia que demuestre beneficios de la colecistectomía a todo paciente con CL como estrategia preventiva en países de baja prevalencia de $\mathrm{CaV}^{32,33}$. Sin embargo, en nuestro medio con elevada mortalidad por esta enfermedad, esta estrategia ha sido propuesta en base a la correlación inversa observada entre la tasa de colecistectomía y la mortalidad por $\mathrm{CaV}$ y a su eventual positiva relación de costo-efectividad ${ }^{34,35}$.

Desde el año 2006, la prevención del CaV ha sido incluida en las Garantías Explícitas en Salud (GES). Inicialmente, la EA y la colecistectomía fueron garantizadas para sujetos portadores o con sospecha de CL sintomática entre 35 y 49 años de edad. A partir del año 2010, esta última fue extendida a todo paciente con CL demostrada dentro del mismo grupo etario independiente de la presencia de síntomas ${ }^{36,37}$.

El presente estudio tiene por objetivo estimar la magnitud de la patología biliar litiásica en Chile y evaluar la cobertura diagnóstica y terapéutica de $\mathrm{CL}$ como estrategia preventiva de $\mathrm{CaV}$, utilizando la información recopilada en la Encuesta Nacional de Salud 2009-2010 (ENS 2009-2010).

\section{Material y Métodos}

\section{Muestra y mediciones}

La ENS 2009-2010 es un estudio de prevalencia realizado en hogares, en una muestra nacional probabilística, estratificada y multietápica de 5.412 personas de 15 y más años. La muestra tiene representatividad nacional, urbano-rural y regional con errores de estimación variables según prevalencias ${ }^{38}$, no superando el $20 \%$ relativo para prevalencias superiores a $4 \%$. Se seleccionó aleatoriamente un adulto al interior del hogar, utilizando el método de Kish ${ }^{39}$. La tasa de respuesta entre los elegibles fue de $85 \%$, con $12 \%$ de rechazo, no contemplándose reemplazos. La aplicación del cuestionario fue realizada por enfermeras entrenadas ${ }^{40}$. Los aspectos bioéticos de la ENS 2009-2010 siguieron las recomendaciones de estudios internacionales de esta naturaleza. El proyecto fue aprobado por el Comité de Ética de Investigación de la Escuela de Medicina de la Pontificia Universidad Católica de Chile.

\section{Definiciones operacionales}

El dolor tipo biliar fue definido a partir de los criterios de ROMA III ${ }^{41,42}$ como aquel dolor de inicio postprandial, ubicado en hipocondrio derecho y/o epigastrio identificados por una imagen abdominal dividida en cuadrantes, de al menos 30 minutos de duración, sin diarrea asociada y presente en forma única o recurrente en los últimos 5 años. Se consideró como EA al auto reporte de realización de EA no obstétricas realizadas en el período 2006-2010, independiente del motivo de indicación y del operador. Además, se interrogó por el hallazgo de cálculos vesiculares en alguna de las EA realizadas. Se consideraron sujetos colecistectomizados a aquellos individuos que reportaron haber sido operados de la vesícula biliar o por cálculos vesiculares.

La ENS 2009-2010 entrega datos sobre Índice de Masa Corporal (IMC kg/m²), en base a medición estandarizada de peso y talla y sobre el nivel de instrucción estratificado en $<8$ años; 8 a 12 y $>12$ años de estudio.

Se obtuvo la mortalidad por $\mathrm{CaV}$ de la base de datos del Departamento de Estadísticas e Información de Salud (DEIS) del año 2010.

\section{Estadística}

El análisis estadístico fue realizado utilizando los factores de expansión obtenidos según el mues- 
treo complejo de la ENS 2009-2010. Los datos se expresan como frecuencia e intervalo de confianza 95\% (IC95\%) o media e IC95\%. Para la asociación de variables se realizaron regresiones logísticas, expresadas según Odds Ratio (OR) e IC95\%. Los estimadores de riesgo por región fueron calculados con la Región Metropolitana (RM) como población de referencia. Se estableció a priori un valor alfa de $5 \%$. Todos los análisis fueron ejecutados a partir del módulo de muestras complejas del paquete estadístico SPSS 17.0.

\section{Resultados}

\section{Dolor tipo biliar y demanda potencial}

\section{de ecotomografía abdominal}

La prevalencia de dolor tipo biliar dentro de los últimos 5 años fue $7,1 \%$ (IC 95\%; 5,9-8,6) en población no colecistectomizada (Tabla 1), con predominio del sexo femenino, OR: $2,7(1,7-4,1)$ $(\mathrm{p}<0,001)$, ajustado por edad. La distribución del dolor tipo biliar según edad se muestra en la Figura 1. La prevalencia de dolor tipo biliar en población colecistectomizada fue $11,1 \%(8,0-15,3)$, sin diferencias con la población no colecistectomizada al ajustar por edad y sexo $(\mathrm{p}=0,27)$.

La distribución regional del dolor tipo biliar se presenta en la Tabla 2. Con respecto a la Región Metropolitana, Coquimbo y La Araucanía presentaron una prevalencia significativamente menor con un OR $0,32(0,1-0,7)$ y OR $0,38(0,1-$ $0,9)$ respectivamente, ajustado por edad y sexo.

No se observaron diferencias en prevalencia de dolor biliar según nivel educacional, zona urbana o rural, estado nutricional o paridad ajustadas por edad y sexo (Tabla 3 ).

Así, mediante la ENS se estimó que en el país existirían 836.796 individuos con antecedente

Tabla 1. Distribución demográfica de dolor tipo biliar, ecotomografía abdominal (2006-2010) y prevalencia de vida de colecistectomía en población de 15 años y más

\begin{tabular}{|c|c|c|c|c|c|c|c|c|c|}
\hline & \multicolumn{3}{|c|}{ Dolor biliar } & \multicolumn{3}{|c|}{ Ecotomografía } & \multicolumn{3}{|c|}{ Colecistectomía } \\
\hline & $\%$ & IC95\% & $\mathbf{n}$ & $\%$ & IC95\% & $\mathbf{n}$ & $\%$ & IC95\% & $\mathbf{n}$ \\
\hline Prevalencia general & 6,3 & $5,2-7,6$ & 298 & 16,2 & $14,6-18$ & 891 & 11,2 & $9,9-12,5$ & 779 \\
\hline \multicolumn{10}{|l|}{ Sexo } \\
\hline Femenino & $8,6^{*}$ & $6,9-10,7$ & 220 & $21,2^{*}$ & $18,8-23,9$ & 648 & $16,6^{*}$ & $14,6-18,7$ & 623 \\
\hline Masculino & 3,9 & $2,8-5,5$ & 78 & 11 & $9-13,4$ & 243 & 5,5 & $4,2-7,1$ & 156 \\
\hline \multicolumn{10}{|l|}{ Edad (años) } \\
\hline$<35$ & 6,6 & $4,7-9,2$ & 94 & $13,4^{*}$ & $11-16,1$ & 246 & $1,1^{*}$ & $0,7-1,8$ & 40 \\
\hline $35-49$ & 7,4 & $5,4-10,0$ & 101 & 18,1 & $14,8-22$ & 240 & $7,6^{*}$ & $5,7-10$ & 133 \\
\hline$>49$ & 5 & $3,7-6,7$ & 103 & 18,1 & $15,4-21,3$ & 405 & 26,9 & $23,8-30,3$ & 606 \\
\hline
\end{tabular}

*Diferencias estadísticamente significativas ajustadas por edad y sexo. IC95\%: Intervalo de confianza 95\%.

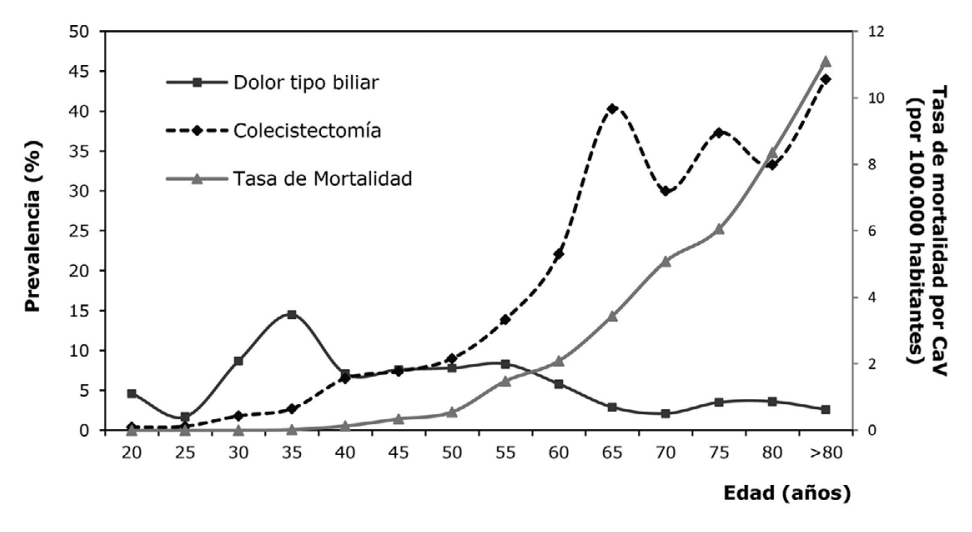

Figura 1. Prevalencia de dolor tipo biliar, colecistectomía y tasa de mortalidad por cáncer vesicular (CaV) al 2010* según edad en población de 15 años y más. *Tasa de mortalidad por CaV obtenida del DEIS. 
Patología de vesícula biliar en la Encuesta Nacional de Salud, Chile 2009-2010 - G. Latorre et al

Tabla 2. Distribución regional de dolor tipo biliar, ecotomografía abdominal (2006-2010) y prevalencia de vida de colecistectomía en población de 15 años y más

\begin{tabular}{|c|c|c|c|c|c|c|c|c|c|}
\hline \multirow{3}{*}{ Región } & \multicolumn{3}{|c|}{ Dolor Biliar } & \multicolumn{3}{|c|}{ Ecotomografía } & \multicolumn{3}{|c|}{ Colecistectomía } \\
\hline & $\%$ & IC95\% & $\mathbf{n}$ & $\%$ & IC95\% & $\mathbf{n}$ & $\%$ & IC95\% & $\mathbf{n}$ \\
\hline & & & & & & & & & \\
\hline XV. Arica y Parinacota & 7,3 & $4,2-12,3$ & 20 & 18,1 & $12,9-24,8$ & 54 & 8,5 & $5,7-12,4$ & 36 \\
\hline I. Tarapacá & 5,3 & $2,7-10,1$ & 12 & 19,9 & $13,3-28,7$ & 56 & 16,6 & $10,3-25,7$ & 49 \\
\hline II. Antofagasta & 4,4 & $2,6-7,2$ & 18 & 17,7 & $13,3-23,2$ & 57 & 11,3 & $8,1-15,5$ & 45 \\
\hline III. Atacama & 5,5 & $3,4-9,0$ & 20 & 15,2 & $10,7-21,3$ & 44 & 12,3 & $8,7-17,8$ & 40 \\
\hline IV. Coquimbo & $2,3^{*}$ & $1,1-4,7$ & 13 & 16,3 & $11,1-23,4$ & 48 & 8,2 & $5,4-12,2$ & 34 \\
\hline V. Valparaíso & 5,6 & $3,2-9,3$ & 22 & 22,0 & $16,8-28,3$ & 77 & 11,6 & $8,4-15,9$ & 53 \\
\hline XIII. Región Metropolitana & 6,7 & $5,0-9,0$ & 57 & 17,2 & $14,1-20,8$ & 151 & 12,2 & $9,9-15,1$ & 127 \\
\hline VI. L. B. O’Higgins & 5,8 & $3,4-9,9$ & 19 & 15,8 & $11,6-21,1$ & 67 & 9,3 & $6,5-13$ & 51 \\
\hline VII. Maule & 6,2 & $3,8-9,9$ & 21 & 13,4 & $9,7-18,3$ & 52 & 12,2 & $8,9-16,3$ & 55 \\
\hline VIII. Bío Bío & 10,3 & $5,7-18$ & 24 & 12,9 & $8,6-18,8$ & 41 & 8,3 & $5,3-12,8$ & 32 \\
\hline IX. Araucanía & $2,6^{*}$ & $1,1-6,1$ & 11 & $6,0^{*}$ & $3,4-10,4$ & 26 & 10,1 & $6,8-14,8$ & 45 \\
\hline XIV. Los Ríos & 4,9 & $2,8-8,7$ & 13 & 16,0 & $11,8-21,3$ & 50 & 18,2 & $13,3-24,4$ & 69 \\
\hline X. Los Lagos & 6,2 & $3,9-9,7$ & 24 & 18,0 & $12,9-24,5$ & 58 & $7,4^{*}$ & $4,9-10,9$ & 39 \\
\hline XI. Aysén & 4,7 & $2,5-8,8$ & 13 & 23,4 & $15,7-33,5$ & 59 & $25,2^{*}$ & $16,3-36,9$ & 49 \\
\hline XII. Magallanes y Ant.Ch. & 4,5 & $1,7-11,5$ & 11 & 15,3 & $8,7-25,5$ & 51 & 7,6 & $5,4-10,7$ & 55 \\
\hline
\end{tabular}

*Diferencias estadísticamente significativas ajustadas por edad y sexo. IC95\%: Intervalo de confianza 95\%.

de dolor tipo biliar, no colecistectomizados, que requerirían una EA como examen diagnóstico. De éstos, 276.159, es decir el 33\% (24,9-42,3), son potenciales beneficiarios GES al encontrarse en el grupo de edad entre 35 y 49 años (Figura 2A y 2B).

\section{Ecotomografías abdominales}

El 26,5\% (24,5-28,6) refirió haber sido estudiado con una EA alguna vez en la vida y el $16,2 \%$ (14,6-18) en los últimos 5 años, independiente de su motivo de indicación (Tabla 1). Ambas con predominio en mujeres, con un OR ajustado por edad de $2,7(2,1-3,4)$ y $2,1(1,6-2,8)(\mathrm{p}<0,001)$ respectivamente. Los sujetos entre 35 y 49 años representan el 32\% de las EA realizadas en los últimos 5 años.

La prevalencia de EA según región de Chile se presenta en la Tabla 2. La Araucanía presenta una menor prevalencia de EA con respecto a la Región Metropolitana, con un OR de 0,3 (0,2-0,6) $(\mathrm{p}<0,001)$, ajustado por edad y sexo.

Según nivel educacional, los individuos con menos de 8 años y de 8 a 12 años de instrucción presentaron una prevalencia menor de EA, con un OR de $0,4(0,3-0,6)$ y $0,6(0,4-0,8)$ respectivamente ( $\mathrm{p}<0,001$ ), ajustados por edad y sexo (Tabla 3 ). No se observaron diferencias según zona urbana o rural al ajustar por edad y sexo.

Del total de EA reportadas en los últimos 5 años el 20,4\% (16,4-25,2) refirió haber recibido el diagnóstico de CL, 22,4\% (17,4-28,3) en mujeres y $16,5 \%(10,3-25,3)$ en hombres, sin diferencias por sexo al ajustar por edad. El hallazgo de CL fue más frecuente en individuos con dolor tipo biliar $(\mathrm{p}=0,04)$ ajustado por sexo y edad (Figura 3$)$. Sin embargo, del total de EA con hallazgo de CL el $15,8 \%(9,1-26,1)$ correspondieron a individuos con dolor tipo biliar y el 84,2\% $(73,9-90,9)$ restante a individuos sin antecedentes de dolor tipo biliar.

\section{Cobertura diagnóstica de colelitiasis}

Sólo 21\% (14,1-30) de la población con antecedente de dolor tipo biliar en los últimos 5 años, no colecistectomizada, refirió haber sido estudiada 


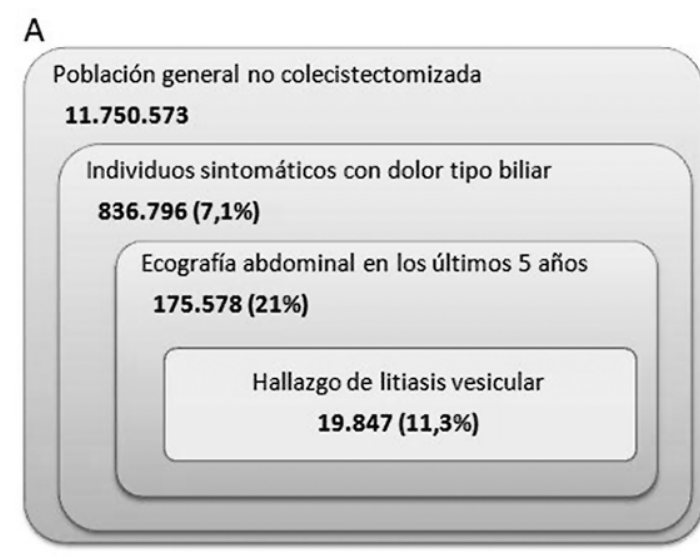

\section{B}

Población entre 35 y 49 años no colecistectomizada

3.744.876

Individuos sintomáticos con dolor tipo biliar

$276.159(7,4 \%)$

Ecografía abdominal en los últimos 5 años $74.715(27,1 \%)$

Hallazgo de litiasis vesicular $2.083(2,8 \%)$

Figura 2. (A) Dolor tipo biliar, ecotomografía abdominal (EA) en los últimos 5 años y hallazgo de litiasis vesicular en población general no colecistectomizada y (B) en población entre 35 y 49 años no colecistectomizada.

Tabla 3. Distribución según nivel educacional de dolor tipo biliar, ecotomografía abdominal (2006-2010) y prevalencia de vida de colecistectomía en población de 15 años y más

\begin{tabular}{|c|c|c|c|c|c|c|c|c|c|}
\hline & \multicolumn{3}{|c|}{ Dolor Biliar } & \multicolumn{3}{|c|}{ Ecotomografía } & \multicolumn{3}{|c|}{ Colecistectomía } \\
\hline & $\%$ & IC95\% & $\mathbf{n}$ & $\%$ & IC95\% & $\mathbf{n}$ & $\%$ & IC95\% & $\mathbf{n}$ \\
\hline \multicolumn{10}{|c|}{ Nivel educacional (años) } \\
\hline$<8$ & 6,7 & $4,6-9,5$ & 69 & $14,3^{*}$ & $11,5-17,5$ & 215 & 24,4 & $20,9-28,3$ & 365 \\
\hline $8-12$ & 5,8 & $4,6-7,2$ & 175 & $14,5^{*}$ & $12,4-16,8$ & 446.230 & 7,7 & $6,4-9,2$ & 303 \\
\hline$>12$ & 7,3 & $4,6-11,4$ & 54 & 21,9 & $17,9-26,6$ & & 9,0 & $6,4-12,3$ & 99 \\
\hline \multicolumn{10}{|l|}{ Zona } \\
\hline Urbana & 6,6 & $5,4-8,1$ & 245 & 16,7 & $14,9-18,7$ & 771 & 11,2 & $9,9-12,7$ & 671 \\
\hline Rural & 4,6 & $3,3-6,4$ & 53 & 13,3 & $10,2-17,0$ & 120 & 10,9 & $8,2-14,3$ & 108 \\
\hline
\end{tabular}

*Diferencias estadísticamente significativas ajustadas por edad y sexo. IC95\%: Intervalo de confianza 95\%.

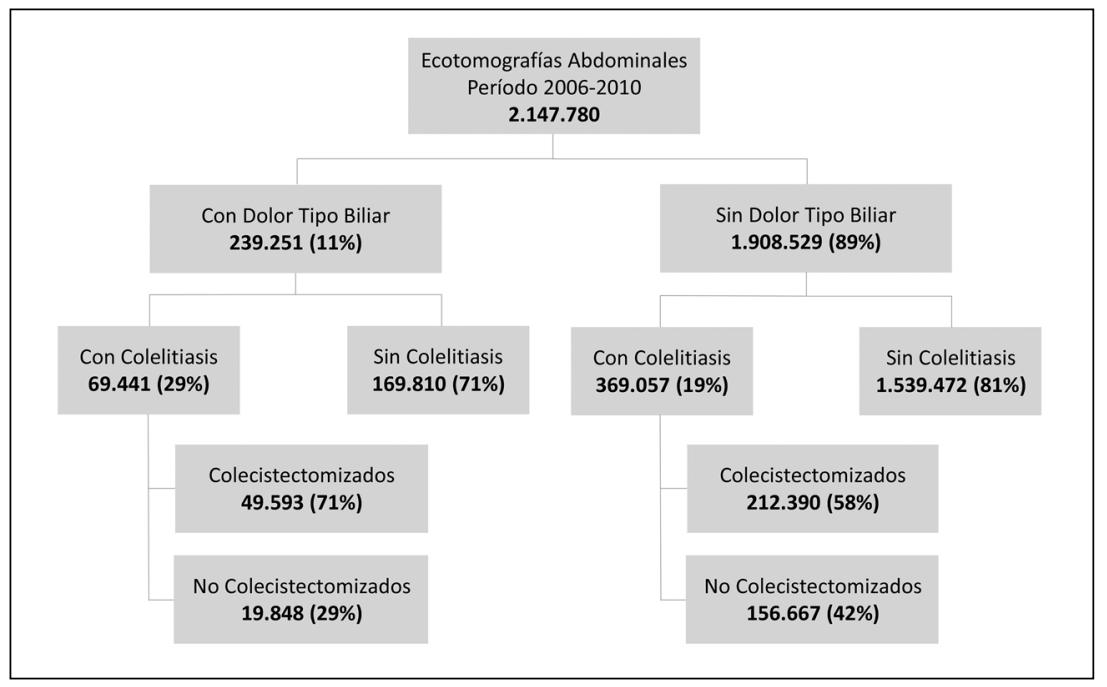

Figura 3. Prevalencia de dolor tipo biliar, hallazgo de colelitiasis y colecistectomía en ecotomografía abdominales realizadas en el período 2006-2010 en población de 15 años y más. 
con una EA (Figura 2A). Así, se estimó una demanda potencial de $661.217 \mathrm{EA}$ a nivel nacional por dolor tipo biliar.

En el grupo de individuos entre 35 y 49 años con dolor tipo biliar, se observó una cobertura de EA de $27,1 \%(15,5-42,9)$, lo que representa una demanda potencial de 201.443 EA para el grupo cubierto por el plan GES (Figura 2B).

No se observaron diferencias según sexo ni por región del país en cobertura de EA. La población de nivel educacional de menos de 8 años presentó una menor cobertura con OR $0,16(0,03-0,8)$ ajustado por edad y sexo $(\mathrm{p}=0,029)$.

\section{Colecistectomías}

La prevalencia observada de colecistectomía en la población mayor a 15 años fue de 11,2\% $(9,9-12,5)$, con un total estimado de 1.478 .760 individuos. Su prevalencia fue mayor en mujeres, con un OR; 3,6 (2,6-5,1) ( $\mathrm{p}<0,001)$ (Tabla 1). Estas representan el 75\% del total de las colecistectomías. La prevalencia de colecistectomía según edad se presenta en la Figura 1.

Según región, Aysén y Los Lagos presentaron prevalencias de colecistectomía con diferencias significativas a la Región Metropolitana, con un OR de 3,2 (1,8-6) y 0,41 (0,2-0,7) respectivamente $(\mathrm{p}<0,001)$, ajustados por edad y sexo (Tabla 2$)$.

No se observaron diferencias según nivel educacional o procedencia urbana o rural ajustados por edad y sexo (Tabla 3 ).

\section{Cobertura de colecistectomía}

El 59,7\% (47,6-70,8) de los individuos que reportan una EA con hallazgo de litiasis vesicular dentro de los últimos 5 años refiere haber sido colecistectomizado (Figura 4), con una cobertura en mujeres de 64,1\%, con un OR 2,9 $(0,9-9,3)$ $(p=0,06)$, sin diferencias significativas al ajustar por edad. Así, se estima una demanda potencial no cubierta de 176.514 colecistectomías a nivel nacional. En el grupo entre 35 y 49 años se observó una cobertura del 56,6\% (30,8-79,3), con una demanda potencial estimada no cubierta de 54.714 colecistectomías, que representan el 31\% (15-53) del total de la demanda potencial para todas las edades.

No se observaron diferencias de cobertura por región o nivel educacional al ajustar por edad y sexo (Figura 4).

En individuos con dolor tipo biliar se obser- vó una cobertura de colecistectomía de 71,4\% $(44,1-88,8)$, en cambio en individuos sin dolor tipo biliar, la cobertura fue de 57,5\% (44,4-69,8) (Figura 3). No se observaron diferencias entre ambos grupos al ajustar por edad y sexo.

\section{Patología biliar litiásica}

A partir de: 1) la prevalencia de colecistectomía; 2) el hallazgo de CL ecográfica en población general y 3) la estimación de CL en población sin EA ni colecistectomía (basado en la proporción de hallazgo de CL en población con EA según la presencia de dolor biliar, Figura 3 ); se estimó que al menos el $26,7 \%$ de la población es o ha sido portador de CL, $36,6 \%$ en mujeres y $16,6 \%$ en hombres.

\section{Discusión}

La CL y $\mathrm{CaV}$ son una importante causa de morbi mortalidad en nuestro país ${ }^{16}, \mathrm{y}$ contribuyen importantemente a posicionar a las enfermedades digestivas como la segunda causa de años perdidos por discapacidad o muerte prematura en Chile ${ }^{43}$. La estrecha relación entre CL y $\mathrm{CaV}$ es un hecho reconocido. A partir del presente reporte, se estimó que en nuestro país al menos $26,7 \%$ de la población ha sido o es portadora de CL. Esta cifra es similar a lo previamente reportado ${ }^{12-16}$. La incorporación de la evaluación mediante la EA en una futura ENS podría esclarecer esta cifra.

Así, considerando que el 7,1\% de la población no colecistectomizada refiere síntomas compatibles con CL, es posible estimar que la mayor parte de la patología biliar se presentaría de forma asintomática, como ha sido demostrado en países desarrollados ${ }^{23,24}$. Además, el bajo reporte de dolor biliar en población con diagnóstico ecográfico de CL (16\%) reafirma la preponderancia de individuos asintomáticos en la presentación de esta patología.

Sin embargo, el presente estudio no permite establecer la sensibilidad y especificidad del dolor tipo biliar en los individuos encuestados. Esto podría influir en la estimación de la magnitud de la CL y la proporción de individuos asintomáticos.

A partir de la ENS es posible estimar una cobertura de $27 \%$ para EA en individuos sintomáticos entre 35 a 49 años, lo cual representa una demanda potencial no cubierta de 201.443 EA para el plan 


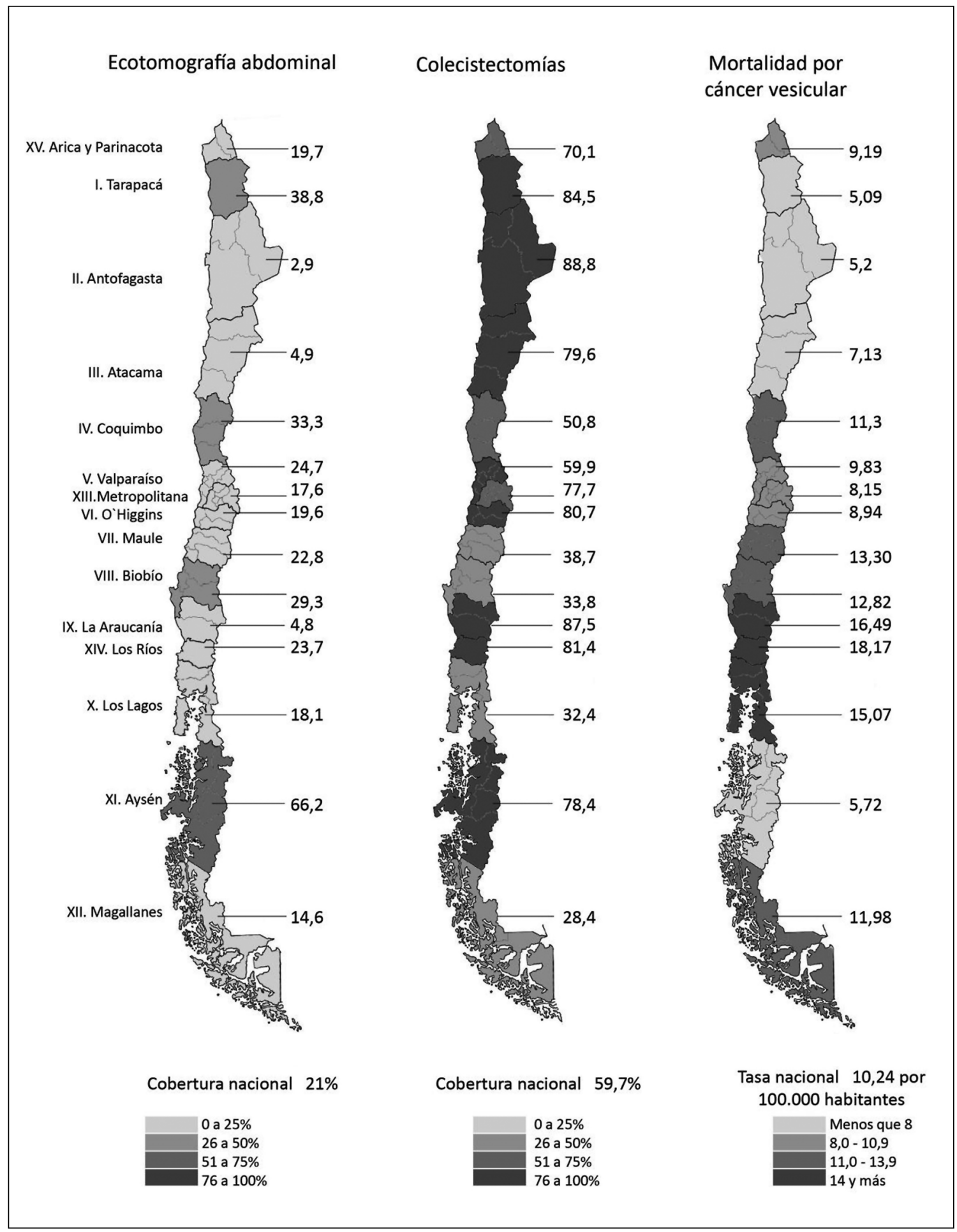

Figura 4. Cobertura de ecotomografía (EA) en individuos con antecedentes de dolor tipo biliar en los últimos 5 años, cobertura de colecistectomías en sujetos con antecedentes EA litiásica y tasa de mortalidad por cáncer vesicular (CaV) del 2010* según distribución por región de Chile en población de 15 años y más. ${ }^{*} T a s a$ de mortalidad por CaV obtenida del DEIS. 
GES. La baja cobertura de esta canasta GES constituye una importante barrera de entrada a esta garantía. Por otra parte, más allá de la garantía de esta prestación, todo dolor tipo biliar debería ser estudiado con una EA independiente de la edad. Según esto, la demanda potencial de EA asciende a 661.217 a nivel nacional.

La cobertura de colecistectomía para portadores de CL entre 35 y 49 años (57\%), permite estimar una demanda potencial no cubierta de 54 mil cirugías. Si se considera que toda CL, independiente de la edad, por su mayor riesgo de desarrollar $\mathrm{CaV}$ u otras complicaciones se beneficia de la colecistectomía, es posible estimar que la demanda potencial no cubierta se extendería a 176 mil colecistectomías a nivel nacional. Más aún, mientras exista una cobertura incompleta de EA, el déficit real de colecistectomías a nivel nacional será subestimado.

Destaca que la prevalencia de ecografía abdominal muestra una inequidad regional y por nivel educacional (menores prevalencias en Araucanía y población con menor nivel educacional), sin embargo, la prevalencia de colecistectomía no mantiene esta tendencia. Esto podría ser explicado por una mayor prevalencia de CL en esta región y en niveles educacionales más bajos.

Si bien la CL sintomática ha demostrado mayor riesgo de $\mathrm{CaV}$, se desconoce aún si los individuos portadores de CL asintomática o silente tienen igual riesgo de desarrollar $\mathrm{CaV}$ que los sujetos sintomáticos en regiones de alta mortalidad por $\mathrm{CaV}$ como es la población chilena. Covarrubias et al. y Csendes et al. ${ }^{14,25}$ han sugerido una historia natural más agresiva de la patología biliar en Chile, con una mayor conversión a formas sintomáticas o complicadas, pero no ha sido demostrado aún si nuestra población con CL asintomática tiene igual riesgo de desarrollar $\mathrm{CaV}$ que la población sintomática. Son necesarios nuevos estudios sobre la historia natural de la CL asintomática y sobre el rol de la colecistectomía preventiva en este grupo en nuestro país.

Independiente del nivel de evidencia del riesgo de desarrollar $\mathrm{CaV}$ en $\mathrm{CL}$ asintomática, el plan GES a partir de 2010 extendió la garantía de colecistectomía para todo individuo portador de CL entre 35 y 49 años de edad, con o sin síntomas al momento del diagnóstico. En este contexto, el presente estudio no demostró diferencias en la cobertura de colecistectomía según la presencia de dolor de tipo biliar, a pesar de la mayor antigüedad de esta garantía en población sintomática.

El objetivo actual de la estrategia preventiva de $\mathrm{CaV}$ del plan GES es obtener una mayor cobertura de colecistectomía a edades más tempranas, previo al aumento de la mortalidad por $\mathrm{CaV}$, lo que en Chile ocurre a partir de la cuarta década de la vida ${ }^{3}$. Sin embargo, según lo expresado en la Figura 1, la prevalencia de colecistectomía en Chile aumenta a partir de los 50 años en adelante. Es esperable que en el futuro, con la estrategia preventiva actual, esto ocurra a edades más tempranas.

Por otra parte, se debe considerar que $90 \%$ de los $\mathrm{CaV}$ incipientes se presentan como hallazgo de colecistectomías en individuos mayores de 49 años $^{44}$. Así, el principal riesgo de la estrategia preventiva actual es excluir de la garantía a aquellos individuos que presentarán lesiones incipientes y curables sólo con la colecistectomía.

No obstante, a pesar de la amplia prevalencia de colecistectomía observada a partir de los 60 años, la mortalidad por $\mathrm{CaV}$ mantiene su aumento. Es posible que una mayor cobertura de colecistectomía no impacte de forma significativa la mortalidad por $\mathrm{CaV}$ en este grupo etario, salvo que sea focalizado en individuos de alto riesgo de $\mathrm{CaV}$. En este contexto, la identificación de factores de riesgo conocidos y por conocer ${ }^{45}$, que permitan predecir el desarrollo de $\mathrm{CaV}$ en población chilena portadora de CL, permitirían priorizar las intervenciones para una mejor estrategia preventiva del $\mathrm{CaV}$.

En conclusión, en nuestro país existe una elevada prevalencia de $\mathrm{CL}$, asociada a una insuficiente cobertura diagnóstica y terapéutica. El indicador más crítico de este problema es la baja cobertura diagnóstica ecográfica, que adicionalmente muestra inequidad regional y socioeconómica. La demanda potencial de prestaciones asociadas a la patología biliar en Chile supera la capacidad de respuesta del sistema de salud, por lo que es necesario establecer criterios técnicos que permitan focalizar las intervenciones en población de mayor riesgo de desarrollar $\mathrm{CaV}$. En este contexto, el futuro descubrimiento de nuevos marcadores de riesgo de $\mathrm{CaV}$ y un mejor conocimiento de la historia natural de la CL asintomática permitirán mejorar la costo-efectividad de las estrategias preventivas aplicadas en Chile.

Agradecimientos: Esta investigación utilizó 
información de la Encuesta Nacional de Salud 2009-2010. Los autores agradecen al Ministerio de Salud, propietario intelectual de la Encuesta, la autorización para disponer de la base de datos. Todos los resultados del estudio son de responsabilidad de los autores y en nada comprometen a dicho Ministerio ${ }^{46}$.

\section{Referencias}

1. Ministerio de Salud de Chile. Estadísticas de mortalidad. Departamento de Estadísticas e Información de Salud, Ministerio de Salud de Chile. Disponible en: http://www. deis.cl. [Consultado el 17 de abril de 2013].

2. Ferlay J, Shin H, Bray F, Forman D, Mathers C, Parkin D. GLOBOCAN 2008 v2.0, Cancer Incidence and Mortality Worldwide: IARC Cancer Base $\mathrm{N}^{\circ} 10$ [Internet]. Lyon, France: International Agency for Research on Cancer; 2010. Disponible en: http://globocan.iarc.fr [Consultado el 25 de julio de 2013].

3. Szot J. Análisis epidemiológico de la mortalidad por tumores sólidos en la Región Metropolitana. Rev Med Chile 2003; 131 (6): 641-9.

4. Henson D, Albores-Saavedra J, Corle D. Carcinoma of the gallbladder. Histologic types, stage of disease, grade, and survival rates. Cancer 1992; 70 (6): 1493-7.

5. Gabrielli M, Hugo S, Domínguez A, Báez S, Venturelli A, Puga M, et al. Letalidad del cáncer de vesícula biliar es independiente del lugar de atención o características socio-demográficas. Chile 2002-2005. Rev Med Chile 2010; 138 (11): 1357-64.

6. Lazcano-Ponce E, Miquel J, Muñoz N, Herrera R, Ferrecio $\mathrm{C}$, Wistuba I, et al. Epidemiology and molecular pathology of gallbladder cancer. CA Cancer J Clin 2001; 51 (6): 349-64.

7. Zatonski W, Lowenfels A, Boyle P, Maisonneuve P, Bueno de Mesquita $\mathrm{H}$, Ghadirian $\mathrm{P}$, et al. Epidemiologic aspects of gallbladder cancer: a case-control study of the SEARCH Program of the International Agency for Research on Cancer. J Natl Cancer Inst 1997; 89 (15): 1132-8.

8. Lowenfels A, Lindstrom C, Conway M, Hastings P. Gallstones and risk of gallbladder cancer. J Natl Cancer Inst 1985; 75 (1): 77-80.

9. Misra S, Chaturvedi A, Misra N, Sharma I. Carcinoma of the gallbladder. Lancet Oncol 2003; 4 (3): 167-76.

10. Butte J, Matsuo K, Gönen M, D’Angelica M, Waugh E, Allen P, et al. Gallbladder Cancer: Differences in Presentation, Surgical Treatment, and Survival in Patients Treated at Centers in Three Countries. J Am Coll Surg
2011; 212 (1): 50-61.

11. Marinovic I, Guerra C, Larach G. Incidencia de litiasis biliar en material de autopsias y análisis de composición de los cálculos. Rev Med Chile 1972; 100: 1320-7.

12. Nervi F, Duarte I, Gómez G, Rodríguez G, Del Pino G, Ferreiro O, et al. Frequency of Gallbladder cancer in Chile, a high risk area. Int J Cancer 1988; 41 (5): 657-60.

13. Roa J, Araya JC, Wistuba J, Villaseca M, Aretxabala X. Litiasis de la vesícula biliar en la IX Región: estudio de autopsias. Rev Med Chile 1991; 119 (12): 1367-71.

14. Covarrubias C, del Pino G, Ferreiro O, Nervi F. Epidemiología e historia natural de la litiasis biliar: implicancias para el manejo clínico de la enfermedad. Rev Med Chile 1992; 120 (4): 432-8.

15. Miquel J, Covarrubias C, Villarroel L, Geltrude M, Greco A, Puglielli L, et al. Genetic epidemiology of cholesterol cholelithiasis among Chilean Hispanics, Amerindians, and Maoris. Gastroenterology 1998; 115 (4): 937-46.

16. Pérez-Ayuso R, Hernández V, González B, Carvacho C, Navarrete C, Álvarez M, et al. Natural history of cholelithiasis and incidente of cholecystectomy in an urban and a Mapuche rural area. Rev Med Chile 2002; 130 (7): 723-30.

17. Medina E, Yrarrázaval M, Kaempffer AM, De Croizer V, Toporowicz M. Epidemiología de las colecistopatías en Chile. Rev Med Chile 1972; 100: 1376-81.

18. Randi G, Franceschi S, La Vecchia C. Gallbladder cancer worldwide: geographical distribution and risk factors. Int J Cancer 2006; 118 (7): 1591-602.

19. Larsson S, Wolk A. Obesity and the risk of gallbladder cancer: a meta-analysis. Br J Cancer. 2007; 96 (9): 145761.

20. Serra I, Yamamoto M, Calvo A, Cavada G, Báez S, Endoh $\mathrm{K}$, et al. Association of chili pepper consumption, low socioeconomic status and longstanding gallstones with gallbladder cancer in a Chilean population. Int J Cancer 2002; 102 (4): 407-11.

21. Dutta U, Garg P, Kumar R, Tandon R. Typhoid carriers among patients with gallstones are at increased risk for carcinoma of the gallbladder. Am J Gastroenterol 2000; 95 (3): 784-7.

22. Friedman G, Raviola C, Fireman B. Prognosis of gallstones with mild or no symptoms: 25 years of follow-up in a health maintenance organization. J Clin Epidemiol 1989; 42 (2): 127-36.

23. Gracie W, Ransohoff D. The natural history of silent gallstones: the innocent gallstone is not a myth. $\mathrm{N}$ Engl J Med. 1982; 307 (13): 798-800.

24. Friedman G. Natural history of asymptomatic and symptomatic gallstones. Am J Surgery 1993; 165 (4): 399-409. 
25. Csendes A, Csendes P, Burdiles P. Seguimiento a largo plazo de pacientes con colecistolitiasis asintomática y sintomática, no operados. Rev Med Chile 1996; 124 (10): 1219-24.

26. De Aretxabala X, Roa I, Araya J, Burgos L, Flores P, Huenchullan I, et al. Operative findings in early forms of gallbladder carcinoma. B J Surg 1990; 77 (3): 291-3.

27. Roa I, De Aretxabala X, Araya J, Villaseca M, Roa J, Guzmán P. Carcinoma incipiente de la vesícula biliar: Estudio clinicopatológico y pronóstico de 196 casos. Rev Med Chile 2001; 129 (10): 1113-20.

28. Braghetto I. Cáncer de la vesícula biliar en la era de la colecistectomía laparoscópica. Rev Chil Cir 1999; 51 (6): 647-54.

29. Waugh E, Friant O, Hamilton J, Caracci M, de la Fuente H, Díaz A, et al. Hospitalización abreviada y colecistectomía abierta electiva. Rev Chil Cir 2000; 52 (2): 159-66.

30. Silva F, Álvarez C, Vergara J, León C, Rojas J. Cáncer de la vesícula biliar en colecistectomías en los hospitales del SSM Sur. Rev Chil Cir 2000; 52 (2): 167-70.

31. Csendes A, Becerra M, Smok G, Medina L, Maluenda F, Morales E. Prevalencia del cáncer de la vesícula biliar en colecistectomías. Rev Med Chile 1991; 119 (8): 887-90.

32. Ransohoff DF, Gracie W, Wolfenson L, Neuhauser D. Prophylactic cholecystectomy or expectant management for silent gallstones. A decision analysis to assess survival. Ann Intern Med 1983; 99 (2): 199-204.

33. Ransohoff D, Gracie W. Treatment of gallstones. Ann Intern Med 1993; 119 (7): 606-22.

34. Chianale J, Valdivia G, Del Pino G, Nervi F. Mortalidad por cáncer vesicular en Chile y su relación con las tasas de colecistectomía. Análisis de la última década. Rev Med Chile 1990; 118 (11): 1284-8.

35. Puschel K, Sullivan S, Montero J, Thompson B, Díaz A. Análisis de costo-efectividad de un programa preventivo de enfermedad vesicular en Chile. Rev Med Chile 2002; 130 (4): 447-59.

36. Ministerio de Salud. Decreto Supremo No 44 Aprueba Garantías Explícitas en Salud del Régimen General de Garantías en Salud de 09-01-2007. MINSAL, Diario Oficial de 31-01-2007. Disponible en: http://www.su- persalud.gob.cl. [Consultado el 16 de enero de 2013].

37. Ministerio de Salud. Decreto Supremo No 04 Aprueba Garantías Explícitas en Salud del Régimen General de Garantías en Salud de 05-02-2013. MINSAL, Diario Oficial de 22-02-2013. Disponible en: http://www. supersalud.gob.cl. [Consultado el 03 de noviembre de 2013].

38. Tabla de errores muestrales absolutos y relativos según prevalencia, ENS 2009-10. Disponible en: http://epi. minsal.cl/estudios-y-encuestas-poblacionales/encuestaspoblacionales/encuesta-nacional-de-salud/resultadosens/ [Consultado el 19 de diciembre de 2012].

39. Kish L. (1965). Survey Sampling. New York, John Wiley \& Sons, Inc.

40. Ministerio de Salud. Informe Encuesta Nacional de Salud 2009-2010, Tomo III- Metodología. Disponible en: http://epi.minsal.cl/estudios-y-encuestas-poblacionales/ encuestas-poblacionales/encuesta-nacional-de-salud/ resultados-ens/. [Consultado el 19 de diciembre de 2012].

41. Behar J, Corazziari E, Guelrud M, Hogan W, Sherman S, Toouli J. Functional Gallbladder and Sphincter of Oddi Disorders. Gastroenterology 2006; 130 (5): 1498-509.

42. Behar J. Physiology and Pathophysiology of the Biliary Tract: The Gallbladder and Sphincter of Oddi-A Review. ISRN Physiology2013; vol. 2013:15. Article ID 837630. doi:10.1155/2013/837630.

43. Minsal (2007) Estudio nacional de carga de enfermedad y carga atribuible a factores de riesgo en Chile. http:// epi.minsal.cl/epi/html/invest/cargaenf2008/Informe $\% 20$ final\%20carga_Enf_2007.pdf

44. Roa I, Muñoz S, Ibacache G, de Aretxabala X. Desde la displasia hasta el cáncer de la vesícula biliar. Rev Med Chile 2009; 137 (7): 873-80.

45. Miquel J (2008). Litiasis biliar. Sociedad Chilena de Gastroenterología (Eds.) Diagnóstico y tratamiento de las enfermedades digestivas. (pp. 371-379). Santiago, Chile. Editorial IKU.

46. Ministerio de Salud, División de Planificación Sanitaria, Departamento de Epidemiología. Disponible en: http:// epi.minsal.cl. [Consultado el 13 de marzo de 2013]. 